Anaesthesist 2017 66:901-903

https://doi.org/10.1007/s00101-017-0382-5

Online publiziert: 13. November 2017

๑) Springer Medizin Verlag GmbH 2017

CrossMark

\title{
U. Schwemmer
}

Klinik für Anästhesiologie und Intensivmedizin, Kliniken des Landkreises Neumarkt, Neumarkt i.d. Opf., Deutschland

\section{Regionalanästhesie: traditio et innovatio}

sorgung fest etabliert. Sie ist $\mathrm{zu}$ einem guten Standard geworden, auf den nicht nur Patienten, sondern auch die operativen Partner in vielen Teilgebieten nicht mehr verzichten wollen. Eingriffe an den großen Gelenken ohne den Einsatz von Schmerzkathetern sind heute kaum noch vorstellbar und sorgen neben der Analgesie für eine frühe Mobilisierbarkeit der Zielstruktur.

\section{Neue Qualität durch Sonographie}

it der zunehmenden Kenntnis der pografischen und funktionellen Anatomie der peripheren Nerven war der Beginn des 20. Jh. auch die Zeit der Entwicklung vieler heute noch etablierter Blockaden, sei es von Nervengeflechten (Plexus) oder von Einzelnerven und den damit verbundenen jeweiligen Zugangswegen. Die meisten Verfahren wurden nach den Erstbeschreibern benannt; eine Tradition, die sich bis ins 21. Jh. aufrecht hielt. Beispielhaft am Plexus brachialis nachvollzogen: Jeder Anästhesist kennt den axillären Zugang nach Hirschel (1911) und den supraklavikulären Zugangsweg nach Kuhlenkampff(1912), den infraklavikulären VIB (vertikale infraclavikuläre Blockade) nach Kilka und Mehrkens (1995), den interskalenären Zugang nach Winnie (1970) und dessen Modifikation nach Meier (1997) [3-7].

Mit der Möglichkeit, Katheter abseits des Epiduralraums an die Zielnerven zu platzieren und so auch postoperativ die Analgesie ohne relevanten Einfluss auf die Vigilanz und die kardiovaskuläre Stabilität weiterzuführen oder auch mit lang wirkenden und sicheren Lokalanästhetika analgetisch zu behandeln, hat sich die periphere Regionalanästhesie über die letzten 20 Jahre in der Patientenver-
Mit der Anwendung der Sonographie für die Nervenblockaden hat die Anästhesie eine neue Qualität in die Patientenversorgung eingebracht. Durch das Erkennen der Anatomie im Ultraschallbild, sowohl der Zielstruktur(en) als auch der Begleitstrukturen, können der optimale Punktionsort und -weg geplant werden. Die meist nach äußeren Landmarken festgelegte Position der traditionellen Blockade wird für den Patienten individuell modifiziert und damit optimiert. Sowohl die Kanüle als auch das Injizieren des Lokalanästhetikums werden sonographisch sichtbar; das Medikament kann präzise an den Ziel-/Wirkort gebracht werden. Durch die Auflösung der Limitation, nach der Detektion des Zielnervs die Ausbreitung des Lokalanästhetikums nicht überwachen und bei Erfordernis durch eine Korrektur der Kanülenposition nachsteuern zu können, wurden die Limitationen der klassischen Nervenblockaden, allen voran die teilweise unzureichende Blockadequalität (inkomplette Blockaden beim Plexus brachii) oder auch höhere Versagerraten, überwunden $[8,9]$. In der Folge haben sich die Ner- venblockaden als Anästhesieverfahren in der Routine weiter etabliert.

Parallel konnte mit der Sonographie auch die experimentelle Forschung zu Fragestellungen der Sicherheit von Nervenblockaden weitervorangetrieben werden. Die Beobachtung von Urmey, dass positive Nervenstimulation und die Parästhesie bei Berührung des Nervs nicht zwingend korrelieren, führte $\mathrm{zu}$ zahlreichen Untersuchungen, die zur Neubewertung der elektrischen Nervenstimulation und zum Verständnis von „Nadel-Nerv-Abstand“ wesentlich beigetragen haben [10-12].

\section{Neue Optionen durch Faszienblockaden}

Die Visualisierung der Begleitstrukturen und damit auch die Darstellung der Begrenzungen anatomischer Räume, in denen sensorische Nerven verlaufen, haben für die Anästhesie von peripheren Nerven weitere Möglichkeiten eröffnet. Mit den Faszienblockaden - der Applikation von Lokalanästhetika zwischen Muskelschichten - wurden in den letzten Jahren viele neue Blockaden beschrieben. Bauchwandblockaden wie der „Transversus-abdominis-plane"(TAP)-Block oder die Blöcke im Bereich der Pektoralismuskulatur (PECS) können definierte Areale anästhesieren und damit neue Aufgabenfelder für den versierten Regionalanästhesisten erschließen. Allerdings gilt es hier kritisch anzumerken, dass gerade für diese vielen neuen Anwendungsbeschreibungen keine wissenschaftlich fundierten Daten zu deren Nutzen für den Patienten, sondern in der Regel nur Fallberichte und Ultraschallbild-Essays existieren. Um aus den 
vielen Ideen dann neue, für die allgemeine Routine des Anästhesisten sinnvolle Blockaden als Standard zu entwickeln, sind prospektive, zielgerichtete Studien notwendig [13].

\section{Bessere Datengrundlage durch Netzwerk Regionalanästhesie}

Ein zweiter, ebenso wichtiger Punkt zur Verbesserung der Qualität in der Regionalanästhesie liegt in der Forschung und wissenschaftlichen Bewertung der klinischen Daten. Es gebührt den Kollegen um Thomas Volk großer Dank und Anerkennung, dass sie mit dem Netzwerk Regionalanästhesie in der DGAI ein zentrales Register geschaffen haben, in dem aus vielen Kliniken die strukturiert erhobenen Daten der Regionalanästhesien gesammelt und nach relevanten Fragestellungen ausgewertet werden. Die Zahl von über 170.000 Fällen, die inzwischen in der Datenbank vorliegen, hat $\mathrm{zu}$ fundierten Ergebnissen und wichtigen Überlegungen zur Verbesserung der Sicherheit, beispielsweise für Epiduralkatheterverfahren durch die Beachtung der Grunderkrankungen und Optimierung der Hygienemaßnahmen, geführt $[14,15]$.

Die wissenschaftliche Arbeit zu Fragen der Regionalanästhesie ist eine zentrale Voraussetzung für die stetige Verbesserung der Versorgungsqualität. Die objektive und qualifizierte Bewertung der Daten und daraus die Entwicklung von Empfehlungen und Leitlinien sind wichtige Arbeiten der Fachgesellschaft, die dafür die Experten in Gremien beruft. Die Arbeitsgruppe Regionalanästhesie hat sich seit mehreren Jahren der Bewertung der Blockaden von oberer und unterer Extremität und der Nervendetektion gewidmet und die Ergebnisse publiziert [16, 17]. Herauszuheben ist die AWMF S1-Leitlinie Handlungsempfehlung zur Nervenlokalisation für periphere Regionalanästhesieverfahren (http://www.awmf.org/leitlinien/detail/ 1l/001-026.html), die auch international Beachtung findet [13].

Wann immer neue Erkenntnisse in der Medizin gewonnen werden, folgen die erforderlichen Änderungen und finden in der klinischen Praxis Einzug. Als
Beispiel kann hier die Entwicklung der Antikoagulantientherapie in den letzten Jahrzehnten dienen. Die Entwicklung von therapeutischen Strategien für die Prophylaxe und auch die Therapie von thrombembolischen Ereignissen hatte auf die Anwendung der rückenmarknahen Regionalanästhesieverfahren relevanten Einfluss. Bereits 1997 publizierte die DGAI die erste Empfehlung zur rückenmarknahen Regionalanästhesie und Thromboseprophylaxe bzw. Antikoagulation [18]. Die Einführung immer neuer Substanzen, zuletzt die direkten oralen Antikoagulanzien (DOAK) und die damit verbundenen Risiken von Blutungskomplikationen resultierten in Erweiterungen und Neufassungen der Empfehlungen [19, 20]. Die Analyse großer Patientenkollektive zeigte die tatsächlichen Risiken bei neuroaxialen Blockaden, insbesondere bei der Anwendung von Epiduralkathetern in bestimmten Patientenkollektiven [21-23]. So existiert für die Regionalanästhesie im Rahmen der Knieendoprothetik mit der Blockade peripherer Nerven heute eine im Vergleich zur Epiduralanästhesie gleich wirksame und sichere Alternative [24].

Der Einsatz der Regionalanästhesie erfolgt in der Regel konsekutiv zur operativen Therapie oder der analgetischen Notwendigkeit, die sich durch einen operativen Eingriff oder ein akutes Schmerzereignis ergibt. Die etablierten zentralen und peripheren Blockaden bieten für die Anästhesie und Analgesie ein großes Leistungsspektrum und lassen nur wenige Lücken. Die meisten Verfahren gelten als Standards, die national und auch international anerkannt sind und vonseiten der Medikation und Durchführung konsentiert sind. Die Überprüfung dieser Festlegungen und eine mögliche Verbesserung, sei es durch den Einsatz neuer Medikamente, Verwendung von Additiva oder neue Techniken, erfordern qualitativ hochwertige und prospektive Studien und die Analyse großer Patientengruppen, wie sie beispielhaft das Netzwerk Regionalanästhesie durchführt. Daneben wird auch weiterhin die wissenschaftliche Auseinandersetzung mit der funktionellen und der topografischen Anatomie, die immer eine zentrale
Grundlage allen regionalanästhesiologischen Denkens bleibt, insbesondere mit der Anwendung des Ultraschalls zu neuen Aspekten der Regionalanästhesie führen. Hier ist exemplarisch die Faszienblockadetechnik zu nennen, die über die direkte Detektion möglicher Areale, die Nervengewebe beinhalten, immer neue Optionen für die Nervenblockade an sehr unterschiedlichen Orten hervorbringt und damit auch neue Indikationsfelder generieren wird.

Standards in der Medizin müssen auf belastbaren Daten fußen und für die grundlegenden Verfahren eine zuverlässige Basis sein. Unter dieser Prämisse ist es wichtig zu erkennen, was wir in der täglichen Arbeit an verlässlichen Methoden benötigen und mit der bestmöglichen Datensicherheit durchführen. Die Forschung auf dem Gebiet der Regionalanästhesie mit all ihren Facetten ist ein Garant für die bestmögliche Qualität unserer Standards und damit auch für unsere medizinische Qualität.

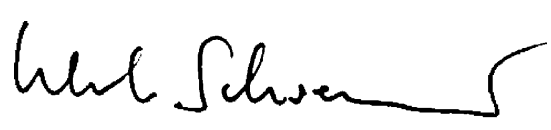

U. Schwemmer

\section{Korrespondenzadresse}

Prof. Dr. U. Schwemmer

Klinik für Anästhesiologie und Intensivmedizin, Kliniken des Landkreises Neumarkt

Nürnberger Str. 12, 92318 Neumarkt i.d. Opf., Deutschland

Ulrich.Schwemmer@klinikum.neumarkt.de

Interessenkonflikt. U. Schwemmer gibt an, dass kein Interessenkonflikt besteht.

\section{Literatur}

1. Goerig M (2015) From the legacy of Carl Koller. Notations on his experiments with cocaine. Anaesthesist 64:469-477

2. Keys TE (1975) Historical vignettes. Anesth Analg $54: 322$

3. Büttner J (1998) Axillary and midhumeral approach for plexus block. Curr Opin Anaesthesiol 11:499-502

4. Tran QH, Clemente A, Doan J, Finlayson RJ (2007) Brachial plexus blocks: a review of approaches and techniques. Can J Anaesth 54:662-674

5. Kilka HG, Geiger P, Mehrkens HH (1995) Infraclavicular vertical brachial plexus blockade. A new method for anesthesia of the upper extremity. An anatomical and clinical study. Anaesthesist 44:339-344 
6. Winnie AP (1970) Interscalene brachial plexus block. Anesth Analg 49:455-466

7. Meier G, Bauereis C, Heinrich C (1997) Interscalene brachial plexus catheter for anesthesia and postoperative pain therapy. Experience with a modified technique. Anaesthesist 46:715-719

8. Abrahams MS, Aziz MF, Fu RF, Horn JL (2009) Ultrasound guidance compared with electrical neurostimulation for peripheral nerve block: a sys tematic review and meta-analysis of randomized controlled trials. Br J Anaesth 102:408-417

9. Lewis SR, Price A, Walker KJ et al (2015) Ultrasound guidance for upper and lower limb blocks. Cochrane Database Syst Rev. https://doi.org/10. 1002/14651858.CD006459.pub3

10. Urmey WF, Stanton J (2002) Inability to consistently elicit a motor response following sensory paresthesia during interscalene block administration. Anesthesiology 96:552-554

11. Sauter AR, Dodgson MS, Kalvøy H et al (2009) Current threshold for nerve stimulation depends on electrical impedance of the tissue: a study of ultrasound-guided electrical nerve stimulation of the median nerve. Anesth Analg 108:1338-1343

12. Vassiliou T, Müller HH, Limberg S et al (2016) Risk evaluation for needle-nerve contact related to electrical nerve stimulation in a porcine model. Acta Anaesthesiol Scand 60:400-406

13. Tran DQ, Boezaart AP, Neal JM (2017) Beyond ultrasound guidance for regional anesthesiology. Reg Anesth Pain Med 42:556-563

14. Bomberg $H$, Kubulus $C$, Herberger $S$ et al (2016) Tunnelling of thoracic epidural catheters is associated with fewer catheter-related infections: a retrospective registry analysis. $\mathrm{Br} J$ Anaesth 116:546-553

15. Bomberg $H$, Kubulus $C$, List F, al German Network for Regional Anaesthesia Investigators et al (2015) Diabetes: a risk factor for catheter-associated infections. Reg Anesth Pain Med 40:16-21

16. Steinfeldt T, Schwemmer U, Volk T, German Society of Anaesthesiology and Intensive Care Medicine (2014) Nerve localization for peripheral regional anesthesia. Recommendations of the German society of Anaesthesiology and intensive care medicine. Anaesthesist 63:597-560

17. Steinfeldt T, Volk T, Kessler P et al (2015) Peripheral nerve blocks on the upper extremity: technique of landmark-based and ultrasoundguided approaches. Anaesthesist 64:846-854

18. Gogarten W, Van Aken H, Wulf H et al (1997) Rückenmarksnahe Regionalanästhesien und Thromboembolieprophylaxe/Antikoagulation. Empfehlungen der Deutschen Gesellschaft für Anästhesiologie und Intensivmedizin. Anasth Intensivmed 38:623-628

19. Gogarten W, Van Aken H, Büttner $\mathrm{H}$ et al (2003) Rückenmarksnahe Regionalanästhesien und Thromboembolieprophylaxe/Antithrombotische Medikation. Überarbeitete Empfehlungen der Deutschen Gesellschaft für Anästhesiologie und Intensivmedizin. Anasth Intensivmed 44:218-230

20. Gogarten W, Vandermeulen E, Van Aken $\mathrm{H}$ et al (2010) Regional anaesthesia and antithrombotic agents: recommendations of the European Society of Anaesthesiology. Eur J Anaesthesiol 27:999-1015

21. Moen V, Dahlgren N, Irestedt L (2004) Severe neurological complications after central neuraxial blockades in Sweden 1990-1999. Anesthesiology 101:950-959

22. Gulur P, Tsui B, Pathak R et al (2015) Retrospective analysis of the incidence of epidural haematoma in patients with epidural catheters and abnormal coagulation parameters. Br JAnaesth 114:808-811

23. Bateman BT, Mhyre JM, Ehrenfeld J et al (2013) The risk and outcomes of epidural hematomas after perioperative and obstetric epidural catheterization: a report from the Multicenter Perioperative Outcomes Group Research Consortium. Anesth Analg 116:1380-1385

24. Gerrard AD, Brooks B, Asaad P et al (2017) Metaanalysis of epidural analgesia versus peripheral nerve blockade after total knee joint replacement. Eur JOrthop Surg Traumatol 27:61-72
Hier steht eine Anzeige. Springer 